Article

\title{
Angiographic Imaging of an In Vivo Mouse Brain as a Guiding Star for Automatic Digital Refocusing in OCT
}

\author{
Kwan Seob Park ${ }^{1,+}$, Ju Wan Kim ${ }^{2,3,+} \mathbb{D}$, Byeong Ha Lee ${ }^{4} \mathbb{D}$ and Tae Joong Eom ${ }^{1, *(D)}$ \\ 1 Advanced Photonics Research Institute, Gwangju Institute of Science and Technology, \\ 123 Cheomdan-gwagiro, Buk-gu, Gwanjgu 61005, Korea; ganseobi@gist.ac.kr \\ 2 Department of Biomedical Science and Engineering, Gwangju Institute of Science and Technology123 \\ Cheomdan-gwagiro, Buk-gu, Gwangju 61005, Korea; scienc2@gist.ac.kr \\ 3 Advanced Instrumentation Institute, Korea Research Institute of Standards and Science, 267 Gajeong-ro, \\ Daejoen 34113, Korea \\ 4 School of Electrical Engineering and Computer Science, Gwangju Institute of Science and Technology, \\ 123 Cheomdan-gwagiro, Buk-gu, Gwangju 61005, Korea; leebh@gist.ac.kr \\ * Correspondence: eomtj@gist.ac.kr; Tel.: +82-62-715-3344 \\ + These two authors have contributed equally to this article.
}

Received: 15 December 2019; Accepted: 3 February 2020; Published: 11 February 2020

\begin{abstract}
A method allows the extraction of the recovery factor that maximizes the image contrast of OCT (optical coherence tomography) and/or OCTA (OCT angiography) of a living subject is proposed in this study. Due to the finite depth of focus in imaging optics, the volume OCT imaging suffers from blurriness in the lateral resolution. By utilizing the digital hologram method or angular spectrum method, the blurred image can be refocused. However, for in vivo OCT imaging, evaluation of the image focus is not easy, owing to the cloudy structure of the brain. In the proposed method, the blood flow signals were used as a guiding star to find the recovery factor. The propagation distance to a focal plane was automatically determined by evaluating the contrast of a cross-sectional OCTA image. The performance was examined though in vivo mouse brain OCT/OCTA imaging. The image singularity of the blood flow in OCTA was very effective at evaluating the contrast of the image.
\end{abstract}

Keywords: digital focusing; optical coherence tomography; optical coherence tomography angiography; brain tissues; blood flow; angular spectrum method; vascular imaging

\section{Introduction}

Optical coherence tomography (OCT) can provide a three-dimensional (3D) structural image of a biological sample [1]. The in vivo, noninvasive, high-resolution image capability of OCT can be useful for early diagnosis or observing the progress of various diseases. OCT has axial and lateral resolutions of several tens of microns that are independently determined by different factors. The axial resolution is proportional to the spectral bandwidth of the light source and the characteristics of the detector. The lateral resolution is determined mainly by the beam diameter and the focal length of the focusing lens, as in light microscopy. Thus, OCT has a trade-off relationship between the transverse resolution and the depth of field (DOF), which might hinder acquisition of high-resolution 3D data for a thick sample. In other words, the transverse resolution of OCT images obtained outside the DOF decreases.

The previous approaches for mitigating the aforementioned problem in OCT imaging are roughly classified into hardware-based dynamic focusing methods and software-based digital focusing methods. The former methods extend the DOF of the system by directly moving the focal plane using various optics, such as a microelectromechanical mirror [2], a liquid-filled polymer lens [3], 
a GRIN lens [4], an acousto-optic tunable lens [5], or an axicon lens [6]. These methods are effective, but are accompanied by increased system cost and decreased imaging speed. On the other hand, the digital focusing method improves the lateral resolution by calculating the field distribution of the image obtained at an out-of-focus region through digital computation without any modification of the system's hardware configuration.

The digital focusing method has been applied for improvement of both axial and lateral resolution by means of deconvolution. The axial deconvolution method has been demonstrated to enhance the depth measurement range by applying dispersion compensation [7]. Likewise, the methods based on spectral shaping have also been demonstrated to enhance the depth resolution of OCT [8]. Similarly, lateral resolution improvements have been demonstrated with the deconvolution methods $[9,10]$, inverse scattering algorithm [11], Gabor-based fusion technique [12], guide-star-based technique [13], scalar diffraction algorithm [14,15], and hybrid hardware and software method [16]. However, most of the aforementioned methods require some prior knowledge of the sample and the imaging system, such as a refractive index of samples, the point spread function (PSF) of the system, and the focal position of the probe beam. To address this, a digital focusing of OCT images without prior knowledge has been demonstrated by automatically finding the distance between the de-focal plane and the focal plane via scalar diffraction theory and information entropy [17]. However, application of this technique to in vivo imaging applications-where the challenges are obvious due to motion artifact-have not yet been demonstrated.

Recently, OCT has been considered as a promising tool for brain imaging, providing 3D structural and vascular images in vivo $[18,19]$. Although the digital focusing method may prove useful for enhancing the depth measurement range in brain OCT imaging, applying the digital focusing method to in vivo brain imaging applications is challenging. Digital refocusing of blurred images may be critical for in vivo brain imaging because the small vessels get easily blurred due to their small size or low contrast. Such digital refocusing has not been demonstrated for in vivo brain imaging so far. To solve this problem, we have utilized the vascular structure of the brain as an image singularity or as a guiding star for the digital refocusing method. However, in the OCT brain images, it is difficult to identify the image singularity. The blood vessel is unclear in an OCT image. Therefore, we used the OCTA (OCT angiography) modality to capture the blood flow that cannot be seen in the OCT intensity images. Since blood vessels are good image singularities in OCTA [20-22], they can act as good guiding stars for automatically refocusing the OCT image.

In this paper, we propose and present a specific method that can be applied for automatic digital refocusing of in vivo brain OCT images. First, we describe the entire methodology for automatically recovering defocused images. After that, the performance evaluation of proposed method by imaging the brain of a mouse in vivo is described. Finally, the brain image refocused automatically by the proposed method is compared to the original image.

\section{Motivation and Methods}

\subsection{Limitation of Depth of Field (DOF) in OCT Imaging}

Figure 1 shows a situation where the DOF of the lens is shorter than the possible imaging depth of an OCT system. In this case, OCT images obtained outside the DOF are laterally blurred. This situation can be avoided by using a lens with low numerical aperture, but this would also worsen the lateral resolution. In Figure 1, the focal plane of the lens is on the corpus callosum in a mouse brain. Since the superficial region of the brain is outside of the DOF, the OCT image blurs laterally. This is apparent for the blood vessels in the OCTA images. Determining some singular points in an OCT image is difficult; therefore, the blurriness of the image cannot be identified easily. However, in the OCTA image when the noisy structures are excluded, we can identify many singular points due to existence of blood flow in a living subject. Therefore, the blood vessel images of OCTA can be used as a guiding star for digital refocusing of the OCT image. 


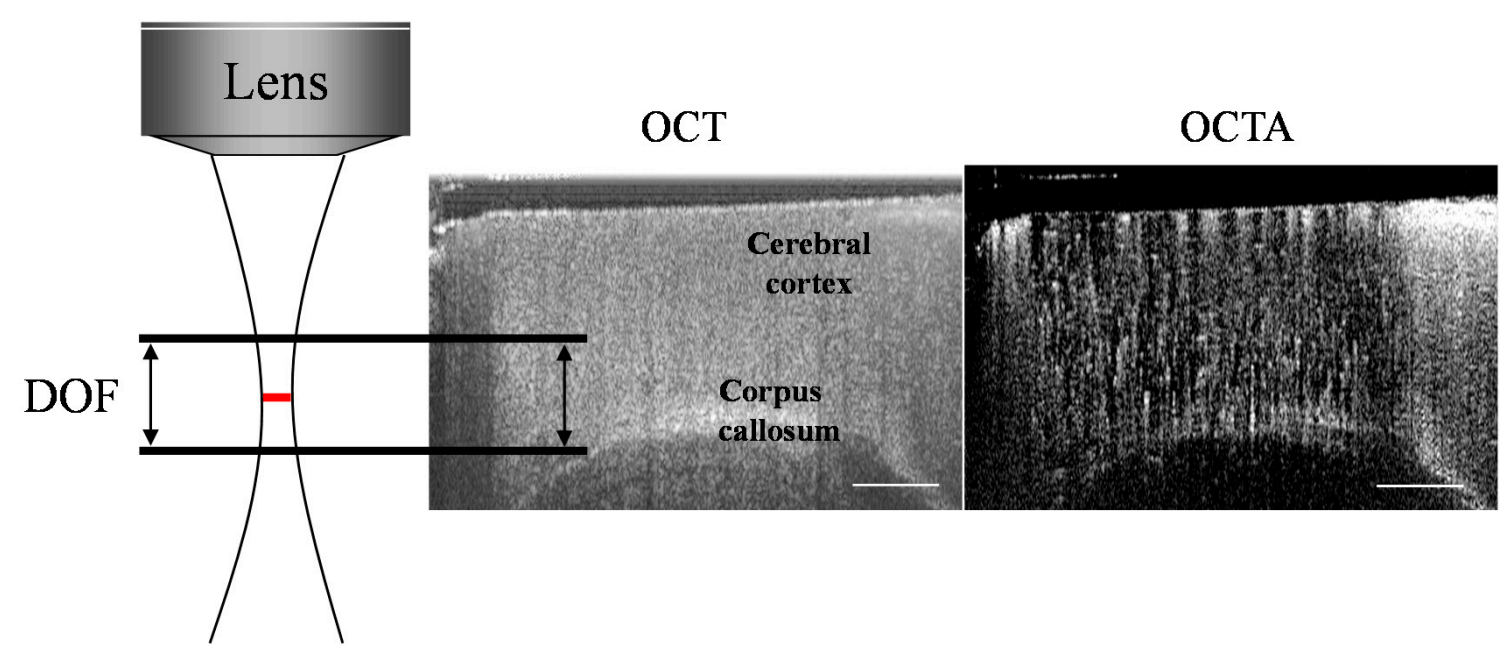

Figure 1. Illustration showing a decrease in lateral resolution for the region outside DOF, on mouse brain imaging using optical coherence tomography (OCT) and OCT angiography (OCTA). Scale bar is $1 \mathrm{~mm}$.

\subsection{Angular Spectrum Method for Digital Focusing of OCTA}

To obtain a volumetric blood microvasculature of a mouse brain, multiple B-scan OCT images were acquired at the same position, and then repeatedly in the C-scan direction. From each B-scan image, the complex OCT signal, denoted by $C(x, z)$, was calculated with a well-known conventional method $[23,24]$. The spectral-domain OCT provides not only a depth resolved OCT image but also the phase information at each depth. A cross-sectional blood flow image was acquired by extracting dynamic signals from four repeated B-scan OCT images using an OCTA technique given by [22].

$$
I_{\text {Flow }(x, z)}=\frac{1}{N-1} \sum_{i=1}^{N-1}\left|C_{i+1}(x, z)-C_{i}(x, z)\right|,
$$

where $C_{i}(x, z)$ is the complex OCT signal in the $i^{\text {th }}$ repeated B-scan, and $N$ is the number of repeated B-scans; in our case, $N=4$.

Since the SD-OCT scheme provides phase information of a subject at all 3D positions, we can use the angular spectrum method (ASM) for simulating wave propagation $[25,26]$. For example, if a lateral plane at certain depth of a 3D subject is out-of-focus upon imaging, we can refocus it using the ASM. Of course, the lateral planes at various depths can have independent refocusing parameters that can be adjusted at every depth of the lateral plane. The digital refocusing based on ASM does not affect the numerical resolution of the system [27]. In the ASM, a complex wave field at a certain depth is decomposed into a sum of a number of plane waves by using a Fourier transform. The decomposed plane waves propagate separately along their directions and recombine at a lateral plane. When the propagation distance of the wave along the $\mathrm{z}$ direction is $d$ (optical distance in air), the complex wave field at another depth is given as $[25,26]$ :

$$
C_{i}(x, z=d)=\mathfrak{J}^{-1}\left\{\mathfrak{J}\left\{C_{0}(x, z=0)\right\} \exp \left[i d \sqrt{k^{2}-k_{x}^{2}}\right]\right\},
$$

where $C_{i}(x, z ; d)$ is the line complex field at the depth of $\mathrm{z}$ after propagating as $d$ along the $\mathrm{z}$ direction, $\mathfrak{I}\left\{C_{0}(x, z=0)\right\}$ is the angular spectrum at the original depth of the 3D OCT data, $k$ denotes the wave number in the medium, and $k_{x}$ is the $\mathrm{x}$ directional wave number. It should be noted that $C_{i}(x)$ corresponds to the line field distribution along the B-scans, in the fast scanning direction. In the 
calculations, the field distribution along the C-scan (thus, y direction) was excluded as the phase along the C-scan was unstable owing to long measurement time (13 s in our case).

To acquire a well-focused field distribution by using Equation (2), it is necessary to determine the propagation distance $d$ which comprises of multiplication of the physical length and the refractive index of the medium. For this, the point spread function (PSF) of the OCT system and the refractive index of the biological tissues must be considered, which are difficult to obtain accurately. Such requirements complicate the implementation of the digital refocusing algorithm. In order to circumvent these requirements, an algorithm that is capable of automatically determining the appropriate propagation distance $d$ was included. While iteratively evaluating the contrast of the digitally refocused OCTA image, the propagation distance $d$ is determined as the value that provides the OCTA image with the highest contrast. In other words, in the OCTA image with the highest contrast, the average of the square of the differences between adjacent A-scans (gradient in the $x$ direction) is maximized. As the contrast evaluation function, the "squared gradient" method was utilized as in [28]:

$$
F_{s q_{\text {grad }}}(d)=\sum_{x} \sum_{z}\left(I_{\text {Flow }}(x+1, z ; d)-I_{\text {Flow }}(x, z ; d)\right)^{2},
$$

where $I_{\text {Flow }}$ is the OCTA signal. This function sums squared differences between adjacent A-lines, making larger gradients exert more influence. Adjacent pixels in images with high-frequency content would have large differences in intensity. This algorithm assumes that the focused image has a higher-frequency content than the defocused images.

\subsection{Data Processing for Automatic Refocusing of OCTA Data}

For digital refocusing, the propagation distance $d$ between the focal plane and the original de-focal plane was determined by evaluating the contrast of B-scan OCTA images. One of the traditional ways to determine the optimal distance is to ascertain the $d$ value that gives the best contrast of the digitally refocused image. To achieve this, the image contrast is evaluated while iteratively entering multiple $d$ values and simulating a wave propagation. However, the OCT image of the mouse brain shows a rather monotonous structure, as shown in Section 3.2, making determination of optimum propagation distance $d$ difficult. To address this problem, B-scan OCTA images were employed in evaluating the contrast comparison. In addition, to find a more reliable $d$ value, a region of interest (ROI; square window) was set inside the B-scan OCTA image. The image with the largest sum of differences of pixel intensities in the B-scan direction ( $\mathrm{X}$ direction) was judged as the image with the largest contrast. Figure 2 shows the digital focusing process to obtain OCTA images with optimal contrast.

1. Four B-scan OCT images were Fourier transformed along the B-scan direction; they were then multiplied with the potential propagation distances (certain $d$ value); lastly, an inverse Fourier transform was performed. This process was repeated over the depths in the ROI while changing the potential propagation distances consisting of an array of the numbers in the range of $-1.5 \mathrm{~mm}$ to $+1.5 \mathrm{~mm}$. This procedure corresponds to the process number 1 in Figure 2 .

2. The digitally propagated four B-scan OCT images were used to create a single OCTA image using Equation (1), and then the contrast of the OCTA image was evaluated using Equation (3). Prior to contrast evaluation, a Gaussian blurring was applied to the OCTA image to remove noise due to speckles. The size of the filter was $1 \times 5 \mathrm{px}$. In this process, it is evaluated only for the OCTA images in the ROI that cover a certain depth (xz direction).

3. Upon repeated execution of the first and the second processes, an optimum propagation distance, $d$-max-which denotes maximum contrast in the OCTA images-was automatically searched and re-entered. Finally, with the found $d$-max, the optimum or the maximum contrast OCT and OCTA images were reconstructed.

4. The same process was performed with a series of the B-scan OCT images along C-scan direction to get a resolution-improved volume OCT/OCTA image set. 


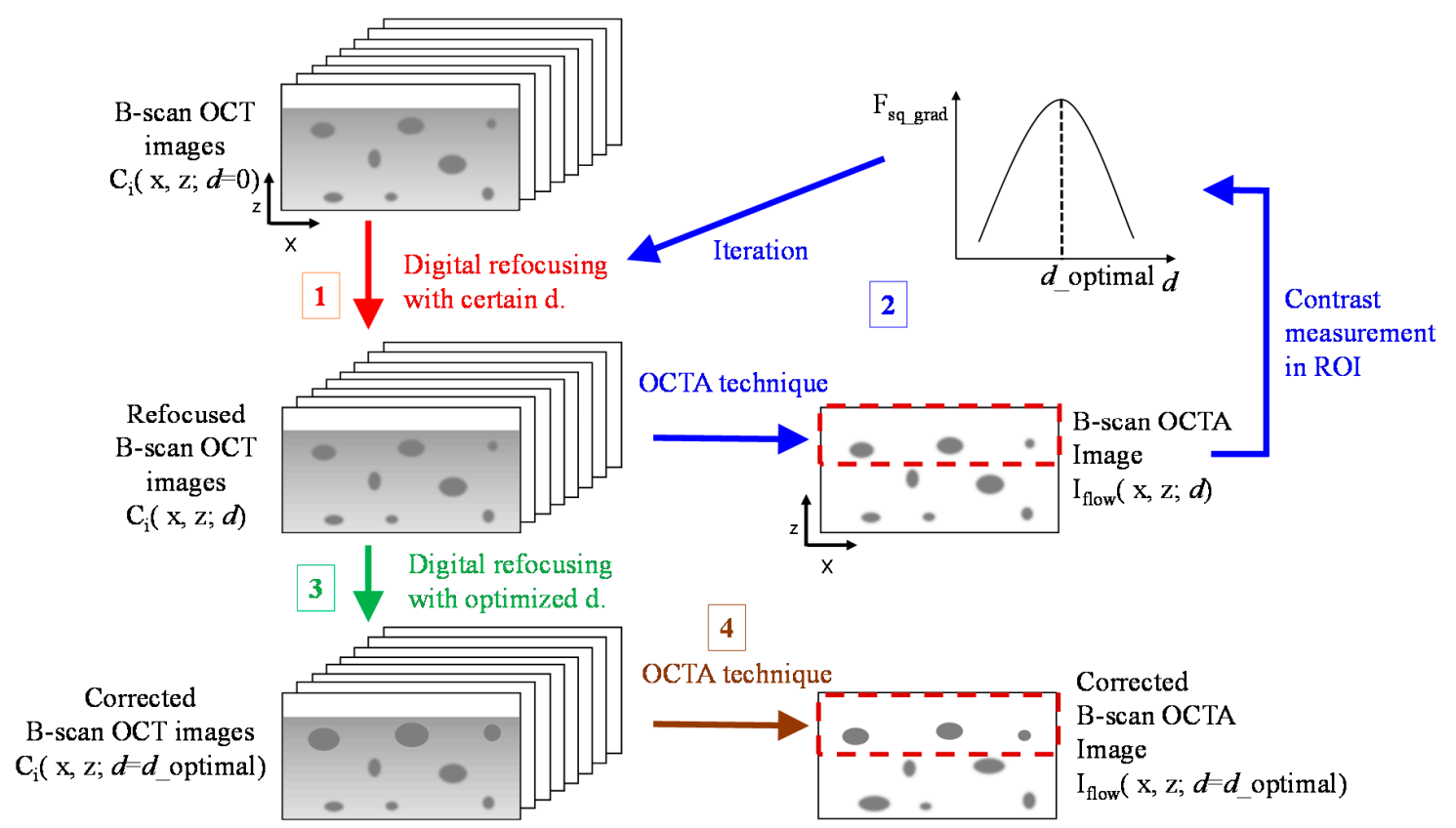

Figure 2. Steps to reconstruct well-focused OCTA images. Complex OCT data are obtained from Fourier transform of the acquired fringes. In the process 2 shown in blue color, the optimal propagation distances, $d$ _optimal, giving the maximum contrast in the OCTA image, are searched for by evaluating the contrast of the OCTA image in the ROI (region of interest) while inserting numerous $d$ values. The round structures in the B-scan OCT images represent vascular structures in the mouse brain.

\subsection{Animal Preparation}

A 7-week-old black mouse (C57BL/6NCrl) with a body weight between 22 and 26 grams was imaged. The mouse was anaesthetized with Zoletil/Xylazine mixture in saline solution $(60 / 10 \mathrm{mg} / \mathrm{kg}$ body weight) during the entire experiment. Craniotomy was performed as defined previously [29]. The cover slip was fixed with the dental acrylic and cyanoacrylate glue. To fix the animal during OCT imaging, a customized angel ring was used for stability, and then the cerebral cortex was imaged. The body temperature was maintained by heating pad at $37^{\circ} \mathrm{C}$ throughout the experiments. In this experiment, all imaging procedures were reviewed and approved by the Institutional Animal Care and Use Committee (IACUC) at the Gwangju Institute of the Science and Technology, South Korea.

\subsection{Spectral-Domain Optical Coherence Tomography for In Vivo Brain Imaging}

Since digital focusing utilizes a complex signal of OCT images, the stable phase of the spectral-domain OCT (SD-OCT) is advantageous for refocusing, and was hence selected for the experiment. As a light source, a superluminescent diode (SLD, DenseLight Semiconductors Incorporated, Singapore) centered at $1310 \mathrm{~nm}$ with a full spectral-width of $150 \mathrm{~nm}$ was used to illuminate the sample, and an interferometric signal was detected by an InGaAs line-scan camera (Sensors Unlimited Corporation, Princeton, NJ, USA) with 2048 pixels, 12-bit resolution, and a maximum line rate of $76 \mathrm{kHz}$. The spectrometer operates in $\mathrm{k}$-space, and does not need a calibration for the wavelength dispersion. The axial resolution of the system was measured to be $8 \mu \mathrm{m}$. The effective imaging range for a $10 \mathrm{~dB}$ sensitivity drop was $3 \mathrm{~mm}$. More details of the system can be found in [30].

\section{Results}

\subsection{Validation of Automatic Refocusing in OCTA Images}

From a set of B-scan OCT images, using the ROI, the OCTA image was extracted by using Equation (1), as can be seen on the right side of Figure 3. The top slice is the OCT image and the slice 
marked 3 is the corresponding OCTA image. With these OCT images, a series of OCTA images were reconstructed by using Equation (2) while changing the propagation distance $d$. The OCTA images reconstructed using another $d$ are depicted in the right side and marked with numbers 1,2 , and 4 . In same manner, the image contrasts of the reconstructed OCTA images were calculated by using Equation (3) and plotted in terms of the propagation distance $d$, as shown in the left graph in Figure 3. The calculated image contrast and the corresponding $d$ values of the four OCTA images on the right side in Figure 3 are indicated with the red arrows in the left graph. The left graph shows the 2nd OCTA slice with the maximum contrast, and the right figure confirms this observation. The blood flow signals in the area marked by the white arrows are clearer than in other images. This signifies that the contrast value can be utilized as a criterion for refocusing of the brain OCT images acquired in vivo.
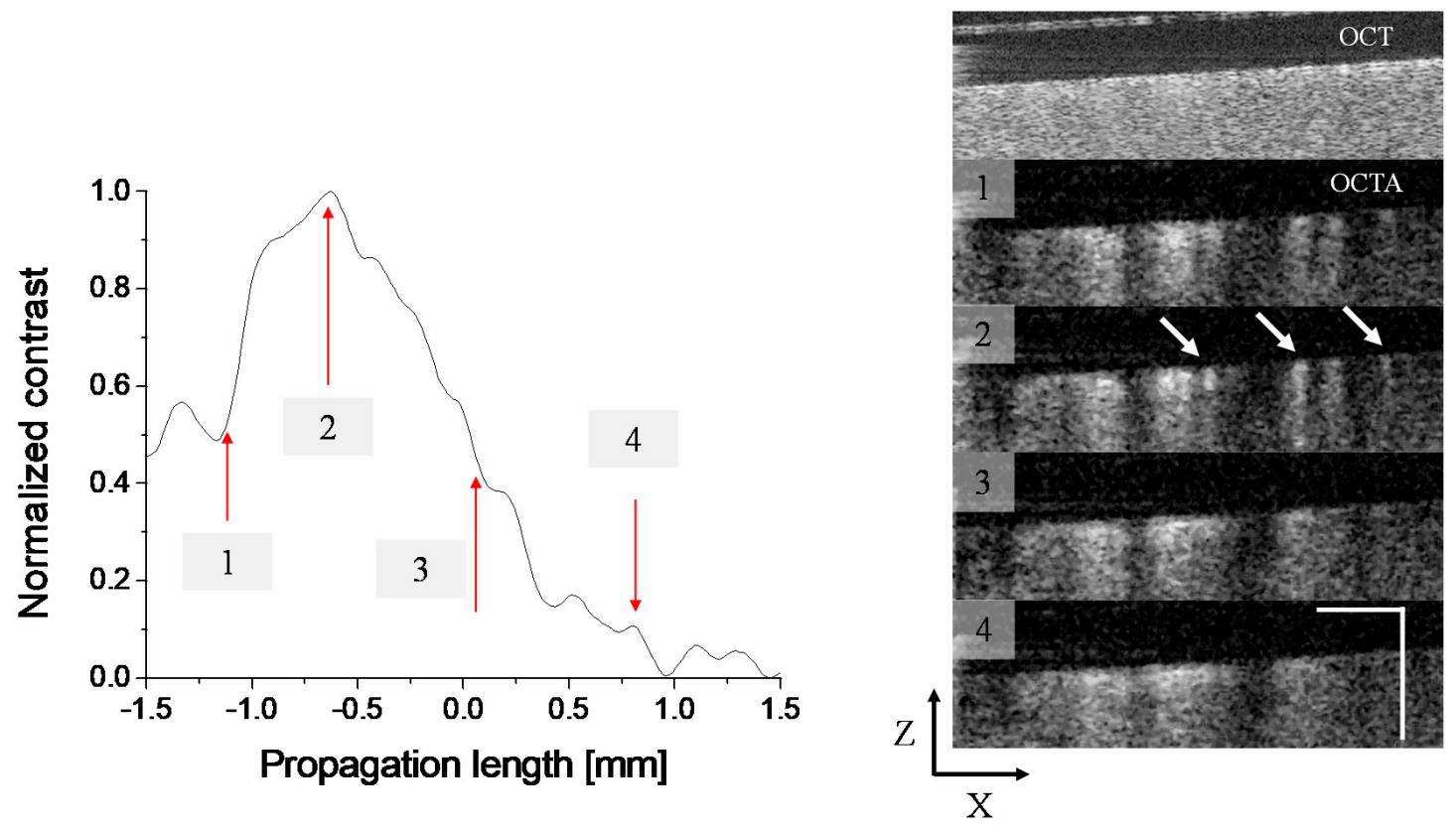

Figure 3. Contrast change of the digitally focused Images 1-4 as a function of the propagation length. Image 2 in the graph shows the highest contrast. White arrows indicate clear flow signals. Scale bar is $500 \mu \mathrm{m}$.

\subsection{Comparison with the Image Refocusing Based on Conventional Structural Images}

In the previous section, the propagation distance $d$ was extracted by evaluating the contrast of the OCTA images and not the conventional OCT images. To confirm the advantage of the proposed method, the image refocusing was performed again by evaluating the contrast of OCT images to predict the optimal propagation distance. With a cross-sectional OCTA image, four different ROIs were established in the depth direction (z direction). In each ROI, the optimum propagation distance was extracted, and with that the OCTA image was reconstructed, as shown in Figure 4a. The middle and right pictures are the ones refocused with the propagation distance $d$ obtained with the OCTA image and the OCT image, respectively. For structure-based recovery, an average of four repeated B-scans was used as a guiding star. Even though the difference is not very clear, the white arrows in the figure show that the blood vessels appear clearly in the middle picture. To quantify the result, the contrast to noise ratio (CNR) of each image was calculated using the metric [31]. The CNR values of the original image, the image reconstructed by the OCTA image, and the image reconstructed by the OCT image were calculated to be $2.8756,3.1146$, and 2.9155 , respectively. 
(a)

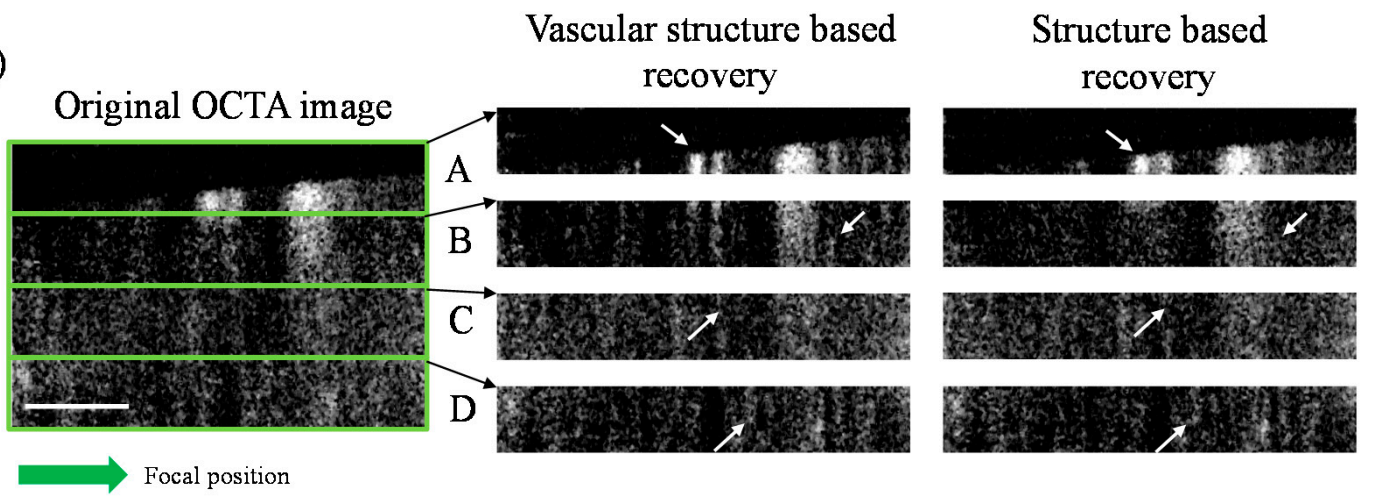

(b)

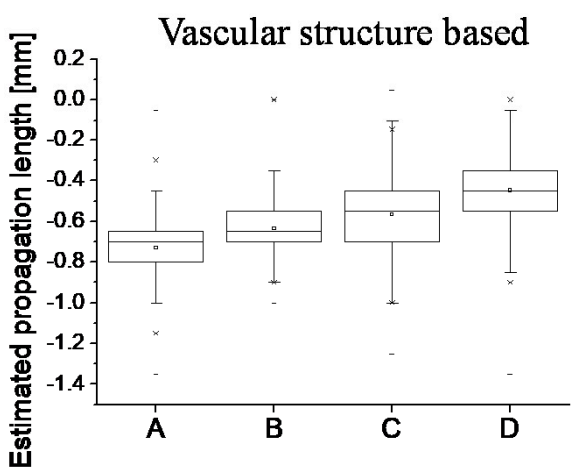

(c)

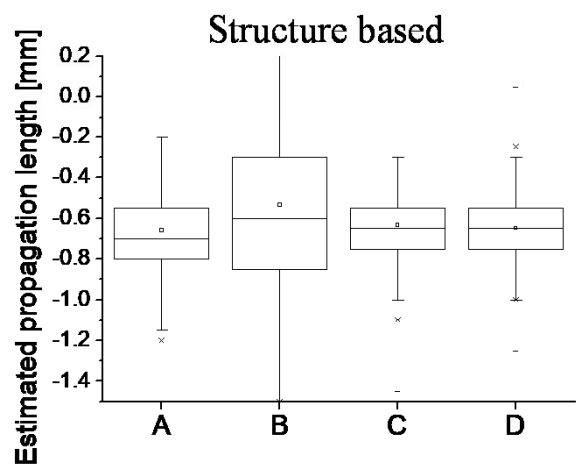

Figure 4. A comparison of the two ways of automatic refocusing of defocused images. (a) Automatically recovered OCTA images by blood flow images and structural images, respectively. White arrows indicate noticeable flow signals between two approaches. Distribution of propagation length estimated by (b) blood flow images and (c) structural images corresponding to each image. The green arrow indicates a focal position. Scale bar is $500 \mu \mathrm{m}$.

To explore further cases, we performed automatic refocusing on 500 defocused OCTA images in the C-scan direction. The statistics of propagation distances calculated for 500 images using OCTA images and OCT images, as guiding stars, were determined, respectively. Figure $4 \mathrm{~b}$ shows the calculated propagation distances, calculated using the OCTA image, shorten as the imaging depth increases (from $\mathrm{A}$ to $\mathrm{D}$ ). The means and standard deviations of the calculated propagation lengths were -0.73 $\pm 0.16,-0.64 \pm 0.14,-0.57 \pm 0.18$, and $-0.45 \pm 0.19 \mathrm{~mm}$ with respect to $\mathrm{A}, \mathrm{B}, \mathrm{C}$, and $\mathrm{D}$, respectively. This makes sense because the deeper the region, the closer the focal plane. The focal position was below the ROI, as indicated using a green arrow in Figure 4a. On the other hand, Figure 4c shows the calculated distances not meaningfully dependent on the depth. The means and standard deviations of the calculated propagation distances were $-0.66 \pm 0.28,-0.53 \pm 0.53,-0.63 \pm 0.26$, and $-0.65 \pm$ $0.16 \mathrm{~mm}$, respectively. Therefore, in conclusion, the refocusing based on the OCTA image is better than the conventional one based on the OCT image. Note that the error bars of Figure $4 \mathrm{~b}, \mathrm{c}$ mean the variations of calculated propagation distances between $500 \mathrm{C}$-scan images, which have slightly different focusing planes.

\subsection{Evaluation in En Face Refocused OCTA Images}

To show the performance of the proposed method more clearly, the contrast of the original (defocused) en face OCTA image was compared with that of the en face OCTA image refocused with the proposed method, as shown in Figure 5. A set of volumetric data, $2048(\mathrm{~A}) \times 500 \times 4(\mathrm{~B} \times$ repetition $) \times$ 500 (C), was acquired while setting the focal plane of probe lens deep inside the mouse brain. A doublet lens with a focal length of $30 \mathrm{~mm}$ was used as a probe lens and the input beam diameters were around 
$2 \mathrm{~mm}$. The theoretical DOF of the system was calculated to be $2.4 \mathrm{~mm}$ in air. Figure $5 \mathrm{a}, \mathrm{c}$ showed en face maximum intensity projections (MIPs) of original and recovered OCTA/OCT images acquired at certain depth ranges. We can see that small vessels and detailed structures indicated as yellow triangles are appreciated in the refocused image. The line profiles along the white dotted lines of Figure $5 a$ were plotted in Figure $5 b$, showing that the OCTA image recovered by using OCTA images as guiding stars has superior contrast in comparison to the original one and the one recovered by using OCT images. Recovered and original OCT images at specific depths $(180,460,740$, and $1020 \mu \mathrm{m})$ are shown in Figure 5c. To quantify the improvement of the image contrast, CNR values of OCT images in Figure $5 \mathrm{c}$ were calculated and plotted in Figure $5 \mathrm{~d}$.
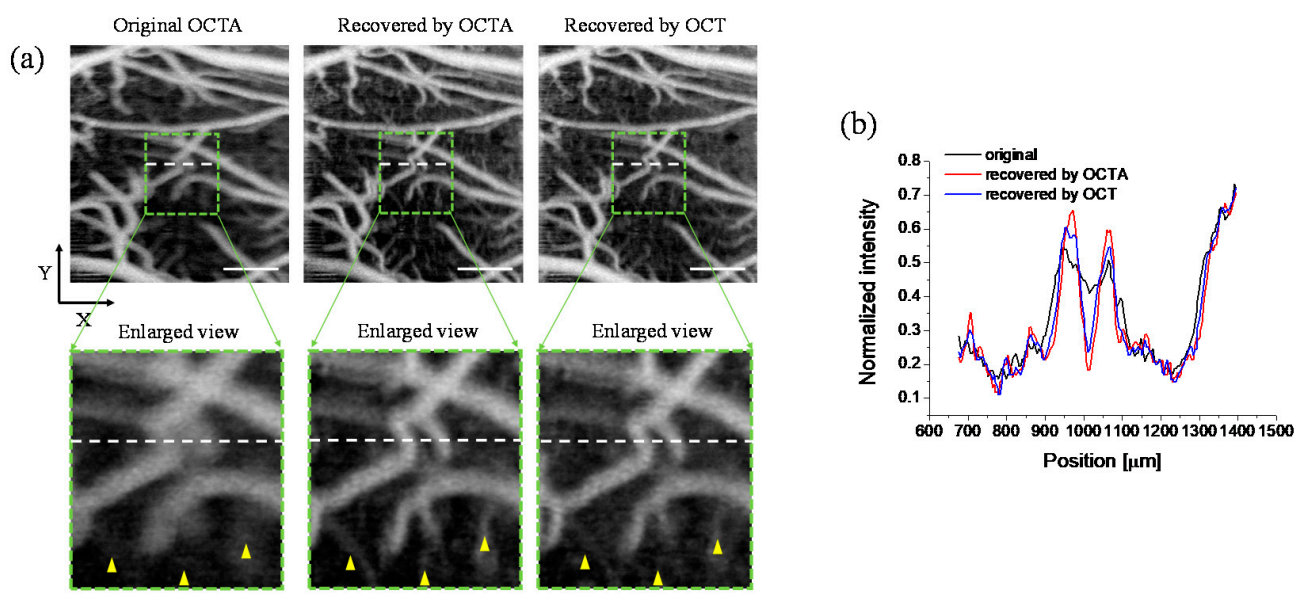

(c)
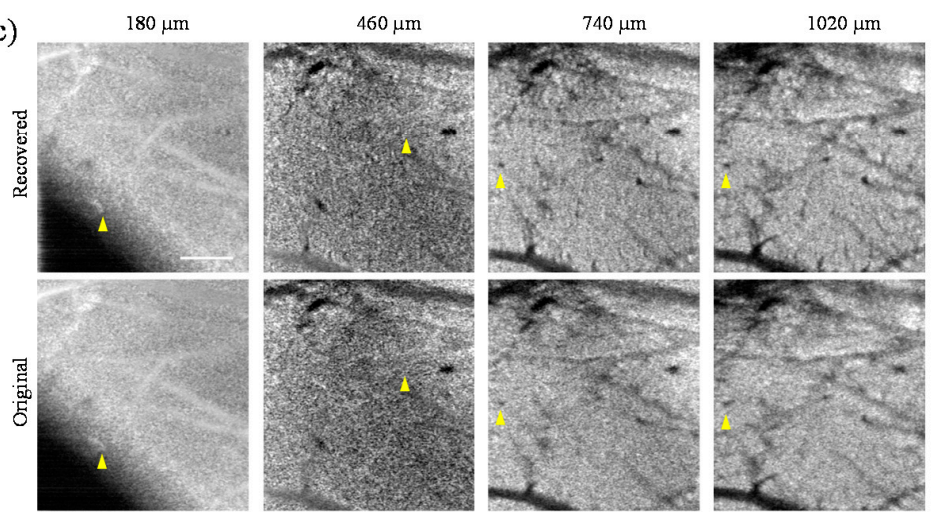

(d)

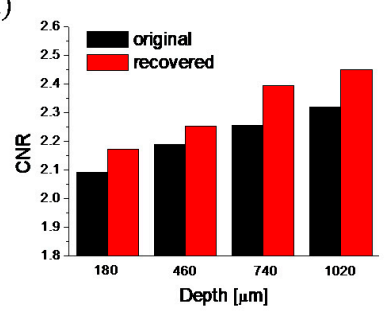

Figure 5. Comparison of en face maximum intensity projections (MIPs) of the original and recovered OCTA/OCT images obtained at specific depths $(0-280 \mu \mathrm{m}$ for OCTA image, and 180, 460, 740, and 1020 um for OCT images). (a) En face MIPs and an enlarged view of original and recovered OCTA images (recovered by OCTA or OCT images), respectively. (b) Line profiles of white dotted lines in (a). (c) Recovered and original OCT images at specific depths. (d) Contrast to noise ratio (CNR) of the OCT images in (c). Yellow triangles show noticeable the contrast change between images. Scale bar is $500 \mu \mathrm{m}$.

\section{Discussion and Conclusions}

In summary, a digital holographic method allowing automatic digital refocusing of an in vivo brain OCT data set obtained at the de-focal plane was demonstrated. An ASM algorithm was utilized to simulate the wave propagation, and the optimum propagation distance was automatically determined by iteratively evaluating the contrast of the digitally focused B-scan OCTA images. Then, the propagation distance providing maximum contrast to the OCTA image was chosen and re-entered to reconstruct the best OCT and OCTA images. The performance of the proposed method was demonstrated by visualizing cross-sectional and en face OCTA images of the mouse brain. 
Furthermore, we have compared the refocusing results using OCT/OCTA images as guiding stars. Using OCTA images demonstrated better performance on the mouse brain tissue. Our processing strategy (using OCTA images as guiding stars) enables software based automatic refocusing with high accuracy in the mouse brain tissue when using SD-OCT for measurements. In this experiment, one dimensional (B-scan direction) focusing was performed. Two dimensional (2D) focusing has not been used due to the instability of the OCT system in the C-scan direction. In future, utilizing another reference arm in the system or a cover glass on top of the sample surface [14] could allow tracing and removal of phase fluctuation along the C-scan direction. 2D refocusing of OCTA images shall be tried.

Author Contributions: Conceptualization, K.S.P. and J.W.K.; methodology, K.S.P. and J.W.K.; software, K.S.P. and J.W.K.; validation, K.S.P., J.W.K., B.H.L., and T.J.E.; formula analysis, K.S.P. and J.W.K.; investigation, K.S.P.; resources, T.J.E.; data curation, K.S.P.; writing—original draft preparation, K.S.P. and J.W.K.; writing一review and editing, T.J.E. and B.H.L.; visualization and review, K.S.P.; supervision, T.J.E.; project administration, T.J.E.; funding acquisition, T.J.E. All authors have read and agreed to the published version of the manuscript.

Funding: This work was supported in part by the "Research on Advanced Optical Science and Technology" grant funded by the GIST, the Industrial Technology Innovation Program, (N0002310, 10063364) of the Ministry of Trade, Industry and Energy of Korea, and a grant of the Korea Health Technology R\&D Project through the Korea Health Industry Development Institute, funded by the Ministry of Health and Welfare (HI18C0858).

Conflicts of Interest: The authors declare no conflict of interest.

\section{References}

1. Huang, D.; Swanson, E.; Lin, C.; Schuman, J.; Stinson, W.; Chang, W.; Hee, M.; Flotte, T.; Gregory, K.; Puliafito, C.; et al. Optical coherence tomography. Science 1991, 254, 1178-1181. [CrossRef] [PubMed]

2. Qi, B.; Phillip Himmer, A.; Maggie Gordon, L.; Victor Yang, X.D.; David Dickensheets, L.; Alex Vitkin, I. Dynamic focus control in high-speed optical coherence tomography based on a microelectromechanical mirror. Opt. Commun. 2004, 232, 123-128. [CrossRef]

3. Divetia, A.; Hsieh, T.-H.; Zhang, J.; Chen, Z.; Bachman, M.; Li, G.-P. Dynamically focused optical coherence tomography for endoscopic applications. Appl. Phys. Lett. 2005, 86, 103902. [CrossRef]

4. Xie, T.; Guo, S.; Chen, Z.; Mukai, D.; Brenner, M. GRIN lens rod based probe for endoscopic spectral domain optical coherence tomography with fast dynamic focus tracking. Opt. Express 2006, 14, 3238-3246. [CrossRef] [PubMed]

5. Grulkowski, I.; Szulzycki, K.; Wojtkowski, M. Microscopic OCT imaging with focus extension by ultrahigh-speed acousto-optic tunable lens and stroboscopic illumination. Opt. Express 2014, 22, 31746-31760. [CrossRef]

6. Ding, Z.; Ren, H.; Zhao, Y.; Nelson, J.S.; Chen, Z. High-resolution optical coherence tomography over a large depth range with an axicon lens. Opt. Lett. 2002, 27, 243-245. [CrossRef]

7. Izatt, J.A.; Kulkarni, M.D.; Wang, H.-W.; Kobayashi, K.; Sivak, M.V. Optical Coherence Tomography and Microscopy in Gastrointestinal Tissues. IEEE J. Sel. Top. Quantum Electron. 1996, 2, 12. [CrossRef]

8. Bashkansky, M.; Battle, P.R.; Duncan, M.D.; Reintjes, J. Signal Processing for Improving Field Cross-correlation Function in Optical Coherence Tomography. Appl. Opt. 1998, 37, 8137-8138. [CrossRef]

9. Yasuno, Y.; Sugisaka, J.-I.; Sando, Y.; Nakamura, Y.; Makita, S.; Itoh, M.; Yatagai, T. Non-iterative numerical method for laterally superresolving Fourier domain optical coherence tomography. Opt Express 2006, 14, 1006-1020. [CrossRef]

10. Ralston, T.S.; Marks, D.L.; Kamalabadi, F.; Boppart, S.A. Deconvolution methods for mitigation of transverse blurring in optical coherence tomography. IEEE Trans. Image Process. 2005, 14, 1254-1264. [CrossRef]

11. Ralston, T.S.; Marks, D.L.; Carney, P.S.; Boppart, S.A. Inverse scattering for optical coherence tomography. J. Opt. Soc. Am. A 2006, 23, 1027-1037. [CrossRef] [PubMed]

12. Rolland, J.P.; Meemon, P.; Murali, S.; Thompson, K.P.; Lee, K. Gabor-based fusion technique for Optical Coherence Microscopy. Opt. Express 2010, 18, 3632-3642. [CrossRef] [PubMed]

13. Adie, S.G.; Shemonski, N.D.; Graf, B.W.; Ahmad, A.; Scott Carney, P.; Boppart, S.A. Guide-star-based computational adaptive optics for broadband interferometric tomography. Appl. Phys. Lett. 2012, 101, 221117. [CrossRef] [PubMed] 
14. Yu, L.; Rao, B.; Zhang, J.; Su, J.; Wang, Q.; Guo, S.; Chen, Z. Improved lateral resolution in optical coherence tomography by digital focusing using two-dimensional numerical diffraction method. Opt. Express 2007, 15, 7634-7641. [CrossRef]

15. Hillmann, D.; Lührs, C.; Bonin, T.; Koch, P.; Hüttmann, G. Holoscopy-holographic optical coherence tomography. Opt. Lett. 2011, 36, 2390-2392. [CrossRef]

16. Liu, S.; Mulligan, J.A.; Adie, S.G. Volumetric optical coherence microscopy with a high space-bandwidth-time product enabled by hybrid adaptive optics. Biomed. Opt. Express 2018, 9, 3137-3152. [CrossRef]

17. Liu, G.; Zhi, Z.; Wang, R.K. Digital focusing of OCT images based on scalar diffraction theory and information entropy. Biomed. Opt. Express 2012, 3, 2774-2783. [CrossRef]

18. Men, J.; Huang, Y.; Solanki, J.; Zeng, X.; Alex, A.; Jerwick, J.; Zhang, Z.; Tanzi, R.E.; Li, A.; Zhou, C. Optical Coherence Tomography for Brain Imaging and Developmental Biology. IEEE J. Sel. Top. Quantum Electron. 2016, 22, 1-13. [CrossRef]

19. Park, K.S.; Shin, J.G.; Qureshi, M.M.; Chung, E.; Eom, T.J. Deep brain optical coherence tomography angiography in mice: In vivo, noninvasive imaging of hippocampal formation. Sci. Rep. 2018, 8, 1-7. [CrossRef]

20. White, B.R.; Pierce, M.C.; Nassif, N.; Cense, B.; Park, B.H.; Tearney, G.J.; Bouma, B.E.; Chen, T.C.; de Boer, J.F. In vivo dynamic human retinal blood flow imaging using ultra-high-speed spectral domain optical Doppler tomography. Opt. Express 2003, 11, 3490-3497. [CrossRef]

21. Leitgeb, R.A.; Schmetterer, L.; Drexler, W.; Fercher, A.F.; Zawadzki, R.J.; Bajraszewski, T. Real-time assessment of retinal blood flow with ultrafast acquisition by color Doppler Fourier domain optical coherence tomography. Opt. Express 2003, 11, 3116-3121. [CrossRef] [PubMed]

22. Wang, R.K.; An, L.; Francis, P.; Wilson, D.J. Depth-resolved imaging of capillary networks in retina and choroid using ultrahigh sensitive optical microangiography. Opt. Lett. 2010, 35, 1467-1469. [CrossRef] [PubMed]

23. Choma, M.; Sarunic, M.; Yang, C.; Izatt, J. Sensitivity advantage of swept source and Fourier domain optical coherence tomography. Opt. Express 2003, 11, 2183-2189. [CrossRef] [PubMed]

24. Leitgeb, R.; Hitzenberger, C.; Fercher, A. Performance of fourier domain vs time domain optical coherence tomography. Opt. Express 2003, 11, 889-894. [CrossRef]

25. Mann, C.J.; Yu, L.; Lo, C.-M.; Kim, M.K. High-resolution quantitative phase-contrast microscopy by digital holography. Opt. Express 2005, 13, 8693-8698. [CrossRef]

26. Yu, L.; Kim, M.K. Wavelength-scanning digital interference holography for tomographic three-dimensional imaging by use of the angular spectrum method. Opt. Lett. 2005, 30, 2092. [CrossRef]

27. Yu, L.; Kim, M.K. Pixel resolution control in numerical reconstruction of digital holography. Opt. Lett. 2006, 31, 897-899. [CrossRef]

28. Peli, E. Contrast in complex images. J. Opt. Soc. Am. A 1990, 7, 2032-2040. [CrossRef]

29. Mostany, R.; Portera-Cailliau, C. A craniotomy surgery procedure for chronic brain imaging. J. Vis. Exp. 2008. [CrossRef]

30. Hyeon, M.G.; Kim, H.-J.; Kim, B.-M.; Eom, T.J. Spectral domain optical coherence tomography with balanced detection using single line-scan camera and optical delay line. Opt. Express 2015, 23, 23079-23091. [CrossRef]

31. Desai, N.; Singh, A.; Valentino, D.J. Practical Evaluation of Image Quality in Computed Radiographic (CR) Imaging Systems; International Society for Optics and Photonics: San Diego, CA, USA, 2010; p. 76224Q.

(C) 2020 by the authors. Licensee MDPI, Basel, Switzerland. This article is an open access article distributed under the terms and conditions of the Creative Commons Attribution (CC BY) license (http://creativecommons.org/licenses/by/4.0/). 